\title{
Three-dimensional colour Doppler of ductus venosus agenesis in the first trimester
}

\author{
D Singh, MD, DNB Radiodiagnosis; L Kaur, MD Radiodiagnosis
}

Prime Diagnostic Centre and Heart Institute, Chandigarh, India

Corresponding author: D Singh (docdivyas@yahoo.co.in)

\begin{abstract}
Ductus venosus (DV) has a pivotal role in the fetal circulation. It serves as a conduit connecting the fetal umbilical and portal venous system with the inferior vena cava. The absence of DV is an uncommon anomaly. In case of agenesis of DV, the umbilical vein joins the fetal systemic venous circulation via the intrahepatic or extrahepatic route. We report a case of absent DV with associated anomaly diagnosed in the first trimester using three-dimensional (3D) colour Doppler.
\end{abstract}

S Afr J Obstet Gynaecol 2016;22(2):65-66. DOI:10.7196.SAJOG/2016.v22i2.1085

DV is an important vascular channel in the fetal circulation. It preferentially channels the oxygenated umbilical venous blood to the vital organs like the brain and the heart. It is routinely evaluated in the first trimester as its abnormality serves as a marker for fetal aneuploidy and cardiac anomalies. Agenesis of DV is a relatively uncommon anomaly. Its prevalence is 1:2 500 between 11 and 14 weeks of gestation. ${ }^{[1]}$ It can be detected in the first trimester using colour Doppler. Our case illustrates the diagnosis of this entity using three-dimensional (3D) colour Doppler.

\section{Case report}

A 27-year-old primigravida attended our institution for firsttrimester screening sonography. Transvaginal sonogram revealed a cystic lesion with septations measuring $3.6 \times 2.4 \mathrm{~cm}$ in the fetal neck, suggestive of a cystic hygroma (Fig. 1A). In addition, there was bilateral pleural effusion with generalised subcutaneous oedema suggestive of hydrops fetalis (Fig. 1B). Colour Doppler sonography revealed the umbilical vein draining into the portal sinus with non-visualisation of the DV, which would connect the portal sinus with the inferior vena cava (IVC) (Figs 1C and 1D). Hence, a diagnosis of agenesis of DV with intrahepatic drainage was made. There were no other associated anomalies. The couple was offered fetal karyotyping. They opted for termination of the pregnancy. Karyotype of the products of conception post termination of the pregnancy revealed 45XO karyotype consistent with diagnosis of Turner's syndrome.

\section{Discussion}

DV performs a crucial role of directing 20 - 30\% of the oxygenated blood coming from the umbilical vein towards the right atrium, and from there through the foramen ovale into the left atrium, and thereafter the brain and the coronary arteries. ${ }^{[2]}$ In the absence of DV, the umbilical venous blood reaches the heart via other routes: intrahepatic or extrahepatic. In the intrahepatic route, the umbilical venous blood drains directly into the portal sinus. From the portal sinus, it reaches the systemic venous circulation either through an abnormal venous channel to the right atrium or via hepatic sinusoids to the hepatic veins. The extrahepatic route bypasses the portal sinus, such that an abnormal venous channel connects the umbilical vein directly to the systemic circulation. This channel may join the right atrium, IVC, renal vein, iliac veins or, less commonly, the left atrium and coronary sinus. ${ }^{[3,4]}$

Agenesis of DV is diagnosed using colour Doppler. The normal DV is visualised in the sagittal section of the fetal abdomen. It is seen beyond the umbilical vein as an area of aliasing in continuity with the portal sinus and draining into the IVC (Figs $2 \mathrm{~A}$ and $2 \mathrm{~B}$ ). On

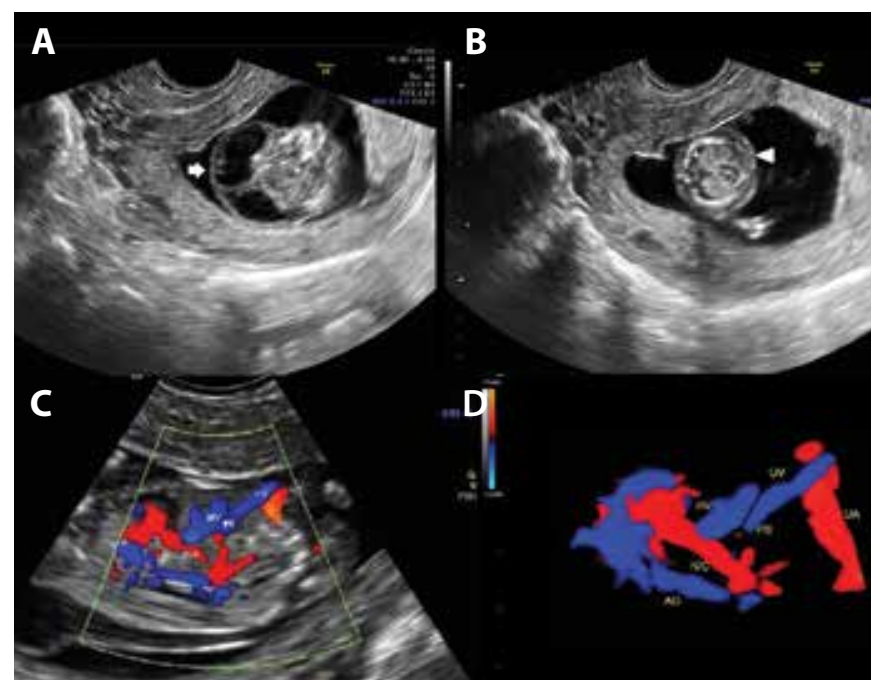

Fig. 1. A. B-mode sonogram of the fetal neck axial section showing an anechoic lesion with septation suggestive of a cystic hygroma (arrow). B. Transverse section of the fetal thorax demonstrating bilateral pleural effusion (asterisk). Note the diffuse oedema of the chest wall (arrow). C. Colour Doppler image of the sagittal section of the fetal abdomen showing the umbilical vein (UV) draining into the portal sinus (PS). No channel is seen connecting the PS to the IVC. These features are suggestive of agenesis of DV. Note the hepatic vein $(H V)$ draining into the IVC. D. $3 D$ colour Doppler image showing the venous connections in the fetus with DV agenesis. (UA = umbilical artery, $A O=$ descending aorta.) 


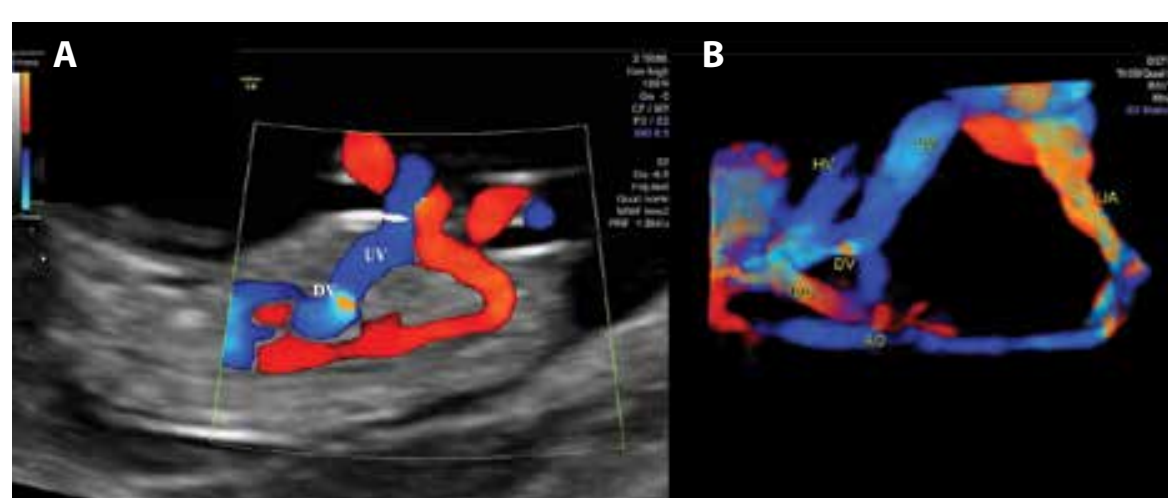

Fig. 2. A. Sagittal colour Doppler image of a normal first-trimester fetus showing the UV connected to the systemic venous circulation by the DV, which is seen as an area of colour aliasing. B. 3D colour Doppler image showing the normal DV.

spectral interrogation, the DV has a triphasic waveform distinct from the monophasic waveform of the umbilical vein. With the recent impetus on first-trimester screening for aneuploidy and availability of highresolution transducers, agenesis of DV can be diagnosed in the first trimester.

The use of $3 \mathrm{D}$ colour Doppler gives a panoramic view of the fetal circulation and helps better understanding of the vascular connections. Thubert et al. ${ }^{[5]}$ reported a case of absent DV in the third trimester using 3D power Doppler. Our case illustrates the 3D colour Doppler findings of DV agenesis in the first trimester. In case of non-visualisation of the DV, a detailed evaluation of the fetal anatomy is mandatory as it may be associated with several anomalies. ${ }^{[6]}$

A recent study demonstrated that in nearly half the cases with absent DV, the fetal nuchal translucency (NT) is above the 95th percentile. In the group with increased NT, more than $40 \%$ of fetuses had chromosomal abnormalities. The most commonly associated aneuploidy was Turner's syndrome. It was observed that in most fetuses with NT below the 95th percentile, absent DV was an isolated finding and these pregnancies had a normal outcome. ${ }^{[1]}$ Therefore, in case of absence of $\mathrm{DV}$ in the first trimester, the prognosis is determined by the NT measurement. If the NT is normal, the prognosis is favourable. In cases with isolated absent DV, the site of umbilical venous drainage is important. Direct drainage of the umbilical venous blood into the right atrium, IVC or its branches can cause volume overload on the fetal heart with the potential to cause cardiomegaly and cardiac failure resulting in hydrops fetalis. ${ }^{[7]}$ Therefore, the prognosis is most favourable for isolated DV agenesis with intrahepatic drainage. Although our case had an intrahepatic drainage of the umbilical vein, it was associated with cystic hygroma and hydrops fetalis. Karyotype of the conceptus revealed Turner's syndrome. This underscores the significance of searching for associated anomalies in agenesis of DV which can determine the prognosis.

In conclusion, agenesis of DV can be diagnosed in the first trimester using colour Doppler. The umbilical venous drainage pathway can be exquisitely depicted using 3D Doppler. The diagnosis of DV agenesis warrants detailed structural evaluation of the fetus for associated abnormalities. Fetal karyotyping should be offered to rule out chromosomal anomalies. If absent DV is seen in isolation, close fetal surveillance is recommended, especially in cases with extrahepatic drainage because of the risk of developing cardiac failure.

\section{References}

1. Staboulidou I, Pereira S, Cruz JdeJ, Syngelaki A, Nicolaides $\mathrm{KH}$. Prevalence and outcome of absence of ductus venosus a 11(+0) to 13(+6) weeks. Fetal Diagn Ther 2011;30(1):35-40

2. Kiserud T. The ductus venosus. Semin Perinatol 2001;25(1):11-20. http://dx.doi.org/10.1053/sper.2001.22896

3. Berg C, Kamil D, Geipel A, et al. Absence of ductus venosus - importance of umbilical venous drainage site. Ultrasound Obstet Gynecol 2006;28(3):275-281. http://dx.doi. org/10.1002/uog.2811

4. Jaeggi ET, Fouron JC, Hornberger LK, et al. Agenesis of the ductus venosus that is associated with extrahepatic umbilical vein drainage: Prenatal features and clinical outcome. Am J Obstet Gynecol 2002;187(4):1031-1037. http://dx.doi.
org/10.1067/mob.2002.126292

.

5. Thubert T, Levaillant JM, Stos B, Benachi A, Picone O. Agenesis of the ductus venosus: Three-dimensional power Doppler reconstruction. Ultrasound Obstet Gynecol 2012;39:118-120. http://dx.doi.org/10.1002/uog.10155

6. Volpe P, Marasini M, Caruso G, et al. Prenatal diagnosis of ductus venosus agenesis and its association with cytogenetic/ congenital anomalies. Prenat Diagn 2002;22(11):995-1000 http://dx.doi.org/10.1002/pd.456

7. Acherman RJ, Evans WN, Galindo A, et al. Diagnosis of absent ductus venosus in a population referred for fetal echocardiography: Association with a persisten portosystemic shunt requiring postnatal device occlusion. J
Ultrasound Med 2007;26(8):1077-1082. 